\title{
VIVÊNCIAS DE ENFERMEIROS E MÉDICOS DE UNIDADES DE PRONTO ATENDIMENTO NO ENFRENTAMENTO DA COVID-19
}

\author{
EXPERIENCES OF NURSES AND DOCTORS \\ OF EMERGENCY CARE UNITS IN \\ COPING WITH COVID-19
}

\section{EXPERIENCIAS DE ENFERMEROS Y MÉDICOS DE UNIDADES DE URGENCIAS EN EL ENFRENTAMIENTO DEL COVID-19}

\author{
Mayckel da Silva Barreto ${ }^{1}$ \\ Sonia Silva Marcon ${ }^{2}$ \\ Anderson Reis de Sousa ${ }^{3}$ \\ Rafaelly de Cássia Nogueira Sanches ${ }^{4}$ \\ Hellen Pollyanna Mantelo Cecilio 5 \\ Dulcineia Martins Pinto ${ }^{6}$ \\ Renata Tresco de Oliveira
}

Como citar este artigo: Barreto MS, Marcon SS, Sousa AR, Sanches RCN, Cecilio HPM, Pinto DM, et al. Vivências de enfermeiros e médicos de Unidades de Pronto Atendimento no enfrentamento da Covid-19. Rev baiana enferm. 2021;35:e43433.

Objetivo: descrever vivências de enfermeiros e médicos de Unidades de Pronto Atendimento no enfrentamento da pandemia da Covid-19. Método: estudo descritivo-exploratório de abordagem qualitativa, realizado com sete médicos e sete enfermeiros atuantes em duas Unidades de Pronto Atendimento, referência para Covid-19. As entrevistas ocorreram entre setembro e novembro de 2020 e foram guiadas por questionário semiestruturado. Os depoimentos foram gravados, transcritos e submetidos a Análise de Conteúdo. Resultados: surgiram duas categorias de análise: "A gente se sente esgotado": a vivência de enfermeiros e médicos e Estratégias para enfrentar os percalços no contexto da pandemia. Considerações finais: os profissionais vivenciaram diversos desafios, como falta de protocolo institucional, falta de estrutura física, material, recursos humanos e capacitação, dificuldade para sensibilizar a população e preocupação de contaminar-se e contaminar a família. Entretanto, apoiaram-se em diferentes estratégias, como autoisolamento preventivo, apoio familiar, troca de experiências com outros profissionais e manter-se atualizado sobre a doença.

Descritores: Infecções por Coronavírus. Pessoal de Saúde. Serviço Hospitalar de Emergência. Percepção Social.

\footnotetext{
Enfermeiro. Doutor em Enfermagem. Docente da Universidade Estadual de Maringá. Maringá, Paraná, Brasil. mayckelbar@gmail.com. https://orcid.org/0000-00032290-8418.

Enfermeira. Doutora em Filosofia da Enfermagem. Docente da Universidade Estadual de Maringá. Maringá, Paraná, Brasil. https://orcid.org/0000-0002-6607-362X.

Enfermeiro. Doutor em Enfermagem e Saúde. Docente da Universidade Federal da Bahia. Salvador, Bahia, Brasil. https://orcid.org/0000-000 I-8534-1960.

Enfermeira. Doutora em Enfermagem. Docente da Universidade Estadual de Maringá. Maringá, Paraná, Brasil. https://orcid.org/0000-0002-I686-7595.

Enfermeira. Doutora em Enfermagem. Docente da Universidade Estadual de Maringá. Maringá, Paraná, Brasil. https://orcid.org/0000-0002-6597-432X

Estudante de Enfermagem. Faculdade de Filosofia, Ciências e Letras de Mandaguari. Mandaguari, Paraná, Brasil. https://orcid.org/0000-000 I-5922-0647.

Estudante de Enfermagem. Faculdade de Filosofia, Ciências e Letras de Mandaguari. Mandaguari, Paraná, Brasil. https://orcid.org/0000-0002-4I23-3682.
} 
Objective: to describe experiences of nurses and doctors of Emergency Care Units in coping with the Covid-19pandemic. Method: descriptive-exploratory study with a qualitative approach, conducted with seven doctors and seven nurses working in two Emergency Care Units, reference for Covid-19. The interviews took place between September and November 2020 and were guided by a semi-structured questionnaire. The statements were recorded, transcribed and submitted to Content Analysis. Results: two categories of analysis emerged: "We feel exhausted": the experience of nurses and doctors and Strategies to face the mishaps in the pandemic context. Final considerations: the professionals experienced several challenges, such as lack of institutional protocol, lack of physical structure, material, buman resources and training, difficulty in sensitizing the population and concern to contaminate oneself and the family. However, they relied on different strategies, such as preventive self-isolation, family support, exchange of experiences with other professionals and keeping up to date on the disease.

Descriptors: Coronavirus Infections. Health Personnel. Emergency Hospital Service. Social Perception.

Objetivo: describir las experiencias de enfermeros y médicos de Unidades de Urgencias en el enfrentamiento de la pandemia de Covid-19. Método: estudio descriptivo-exploratorio con abordaje cualitativo, realizado con siete médicos y siete enfermeros que trabajan en dos Unidades de Urgencias, referencia para Covid-19. Las entrevistas tuvieron lugar entre septiembre y noviembre de 2020 y se guiaron por un cuestionario semiestructurado. Las declaraciones fueron grabadas, transcritas y sometidas a Análisis de Contenido. Resultados: surgieron dos categorías de análisis: "Nos sentimos agotados": la experiencia de enfermeros y médicos y Estrategias para enfrentar los percances en el contexto de la pandemia. Consideraciones finales: los profesionales experimentaron varios desafios, como la falta de protocolo institucional, la falta de estructura física, material, recursos bumanos y capacitación, la dificultad para sensibilizar a la población y la preocupación por contaminar a si mismo y a la familia. Sin embargo, se apoyaron en diferentes estrategias, como el autoaislamiento preventivo, el apoyo familiar, el intercambio de experiencias con otros profesionales y mantenerse al dia sobre la enfermedad.

Descriptores: Infecciones por Coronavirus. Personal de Salud. Servicio de Urgencias Hospitalarias. Percepción Social.

\section{Introdução}

$\mathrm{Na}$ atual conjuntura, o mundo vê-se amplamente afetado pela pandemia ocasionada pelo Severe Acute Respiratory Syndrome Coronavirus-2 (SARS-CoV-2), novo coronavírus, causador da Covid-19 (Coronavirus Disease-19) ${ }^{(1)}$. No Brasil, a pandemia descortinou situações pregressas e históricas de desmonte e negligência das políticas públicas, incluindo o limitado financiamento do Sistema Único de Saúde (SUS), da ciência, das universidades públicas e centros de pesquisa. Ademais, evidencia-se, ainda, a desvalorização do trabalho e dos trabalhadores, especialmente no âmbito da saúde ${ }^{(2)}$.

Sabidamente, durante o avanço acelerado da pandemia da Covid-19, os sistemas de saúde necessitaram reorganizar o atendimento, tendo em vista a alta e crescente demanda. No que tange à organização regionalizada e descentralizada do SUS, além da abertura de novos leitos de Unidade de Terapia Intensiva (UTI) em hospitais gerais, também houve remanejamento da ordenação do fluxo dos pacientes dentro das Redes de Atenção à Saúde. A Unidade de Pronto
Atendimento (UPA), principal componente fixo de urgência e unidade intermediária entre a atenção primária e urgência hospitalar, configurou-se como referência para o atendimento exclusivo a pessoas com suspeita ou confirmação de contaminação pelo vírus ${ }^{(3)}$.

Até março de 2021, período classificado como o pior da pandemia, com recordes diários de mortes, as UPAs, UTIs e enfermarias de hospitais públicos e privados vivenciavam um colapso com falta de leitos e falta de medicamentos e insumos, levando à morte diversos pacientes, ainda na fila de espera. Diante desse cenário caótico, observa-se que a pandemia da Covid-19 tem sido um evento desgastante, traumático e exacerbado para os profissionais atuantes na linha de frente, desencadeando entre eles adoecimento pela exaustão física e mental e mortes em decorrência da contaminação pelo vírus ${ }^{(4)}$. Dessa forma, os profissionais de saúde têm sido vistos como segunda vítima da pandemia da Covid-19 ${ }^{(4)}$.

Analogamente, estudos realizados com profissionais de saúde atuantes em unidades de 
emergência durante outros surtos epidêmicos de novos vírus respiratórios, como Gripe $\mathrm{H} 1 \mathrm{~N} 1^{(5)}$, Síndrome Respiratória Aguda Grave ${ }^{(6)}$ e a Síndrome Respiratória do Oriente Médio por Coronavírus ${ }^{(7)}$, demonstraram que muitos vivenciaram severo estresse no trabalho e esgotamento físico e mental. Por medo da contaminação individual e familiar, alguns profissionais se recusaram a cuidar dos pacientes e abandonaram seus empregos ${ }^{(5-7)}$.

Ressalta-se que o medo e a apreensão constantes entre os profissionais de saúde relacionam-se principalmente ao risco de se expor ao vírus e à preocupação de contágio de suas famílias ${ }^{(8)}$. Portanto, é importante que gestores de UPAs estejam atentos ao estresse profissional associado às experiências de um surto de doenças infectocontagiosas emergentes. Isso porque, nesse momento, os profissionais enfrentam desafios de diversas ordens, incluindo falta de informação, de acesso a Equipamentos de Proteção Individual (EPI), bem como excessiva e exaustiva carga laboral e medo da contaminação ${ }^{(9)}$.

Compreendendo que o trabalho em UPA, referência para pacientes com Covid-19, tem repercutido sobre a vida dos profissionais de saúde, busca-se proporcionar informações para o planejamento de intervenções que se proponham a prevenir ou diminuir o nível de estresse profissional, bem como preparar o sistema de saúde para apoiar os profissionais em futuros surtos de doenças infecciosas de caráter epidêmico ou pandêmico ${ }^{(10)}$. Dessa forma, questiona-se: Quais as vivências de enfermeiros e médicos de UPA no enfrentamento da pandemia da Covid-19? Assim sendo, este estudo tem o objetivo de descrever as vivências de enfermeiros e médicos de UPA no enfrentamento da pandemia da Covid-19.

\section{Método}

Estudo descritivo-exploratório, de abordagem qualitativa, sustentado pela análise de conteúdo e descrito conforme recomendações do Consolidated Criteria for Reporting Qualitative Research (COREQ). Participaram do estudo sete enfermeiros e sete médicos atuantes em duas UPAs, localizadas em dois municípios no estado do Paraná, Brasil, cada qual com população aproximada de 35 mil habitantes. Cada município possui uma UPA, instituição pública que atende pacientes de forma ininterrupta como porta-aberta, sendo a única unidade de emergência referência para os pacientes com sinais e sintomas da Covid-19 dos municípios pesquisados.

A oferta da assistência em ambas as UPAs foi organizada por meio de serviços sentinelas, estruturalmente, separando os pacientes sintomáticos dos demais. A equipe era composta por dois médicos, dois enfermeiros e sete técnicos em enfermagem por período, e trabalhavam em esquema de rodízio entre o Pronto Atendimento Covid (PA-Covid) e o convencional, apoiados por profissionais das Unidades Básicas de Saúde (UBS).

No período da coleta, iniciada em 28 de setembro de 2020, o primeiro município pesquisado apresentava 2.273 casos notificados, com 471 casos confirmados e 8 óbitos devido a Covid-19; o segundo, apresentava 1.916 casos notificados, com 594 confirmações e 7 óbitos.

A amostragem, constituída por conveniência, foi composta ao final por 14 participantes que cumpriram os seguintes critérios de inclusão, ser enfermeiro(a) assistencial ou médico(a) plantonista e atuar há mais de um ano na UPA, o que permitia conhecer o processo de trabalho e a rotina da unidade antes do período pandêmico. Foram excluídos os profissionais (9) que estavam afastados por férias, licença médica e licença maternidade durante o período de coleta de dados.

A abordagem dos participantes foi feita pessoalmente, momento em que se explicou os objetivos da pesquisa e as formas de participação. Diante do aceite, foi agendado horário para entrevista individual, realizada em local reservado dentro das próprias UPAs. Duas acadêmicas de Enfermagem do último período, previamente treinadas, conduziram a coleta de dados de forma conjunta. Destaca-se que elas conheciam a unidade por terem realizado estágio curricular no setor.

A coleta de dados deu-se por meio de entrevista individual aberta, utilizando-se formulário 
4

Vivências de enfermeiros e médicos de Unidades de Pronto Atendimento no enfrentamento da Covid-19

semiestruturado, construído pelos pesquisadores, não validado, sem teste piloto, composto por duas partes: a primeira, com questões sociodemográficas relativas ao sexo, idade, formação acadêmica, tempo de formação, tempo de atuação em unidade de emergência, renda familiar, raça/cor e estado civil; e a segunda, composta por questões de apoio e da seguinte questão norteadora: Fale-me sobre a sua vivência profissional na unidade de pronto atendimento, no contexto da pandemia da Covid-19. As entrevistas tiveram duração média de 35 minutos, foram audiogravadas, permitindo a posterior transcrição na íntegra dos depoimentos. A saturação dos dados foi verificada quando os depoimentos apresentaram consistência e densidade para análise, e os novos dados analisados não agregavam ou aprofundavam as categorias temáticas já elaboradas.

Os dados foram submetidos à Análise de Conteúdo, modalidade temática, empregando-se as etapas de pré-análise, exploração do material e tratamento dos resultados obtidos. $\mathrm{Na}$ pré-análise, as entrevistas foram organizadas e, em seguida, leituras flutuantes permitiram a observação da relação entre o conteúdo obtido e os objetivos propostos. Na exploração do material, as falas foram fragmentadas, aplicando-se distintas cores. Posteriormente, os fragmentos foram agrupados por similaridade semântica em categorias mais abrangentes, as quais consistiram na classificação dos elementos segundo suas semelhanças e diferenças, com posterior reagrupamento, em função de características comuns. $\mathrm{Na}$ última etapa, após a saturação das categorias preliminares, deu-se o tratamento dos resultados, procedendo-se a inferência e a interpretação, com base na literatura específica sobre o tema ${ }^{(11)}$. Esse exaustivo processo analítico levou à identificação de duas categorias temáticas: "A gente se sente esgotado": a vivência de enfermeiros e médicos e Estratégias para enfrentar os percalços no contexto da pandemia.

O projeto de pesquisa foi analisado e aprovado pelo Comitê Permanente de Ética em Pesquisa com Seres Humanos, da Universidade Estadual de Maringá, recebendo o Parecer n. 2.759.729. Os participantes foram esclarecidos quanto ao objetivo do estudo e seus direitos, conforme a Resolução n. 466/12, do Conselho Nacional de Saúde, e suas complementares e assinaram o Termo de Consentimento Livre e Esclarecido (TCLE) em duas vias de igual teor. Para garantir o sigilo e o anonimato, na identificação das falas foram utilizados codinomes constituídos pela letra "E" de Enfermeiro e "M" de médico, seguido de um número arábico referente à ordem de realização das entrevistas.

\section{Resultados}

Participaram do estudo 14 profissionais de saúde, sendo 7 enfermeiros e 7 médicos, cujas idades variaram de 24 a 48 anos, 8 eram do sexo masculino e a maioria (13) de cor branca. Entre os entrevistados, 13 possuíam curso de especialização e atuavam há mais de dois anos na UPA. A experiência profissional variou de 1 a 11 anos (média de 5 anos).

Conforme a análise dos dados, as categorias que foram construídas serão discutidas a seguir.

\section{Categoria temática 1: "A gente se sente esgotado": a vivência de enfermeiros e médicos}

Os participantes revelaram que, entre os principais desafios enfrentados no início da pandemia, estava o fato de a Covid-19 ser uma doença nova, com curso clínico e epidemiológico incerto, o que dificultou o diagnóstico precoce e correto, bem como o tratamento. Além disso, a estrutura das instituições de saúde nos municípios de pequeno porte era incipiente para as altas demandas da pandemia, incluindo a indisponibilidade de testes para diagnóstico em larga escala.

Somado a esse desafio, os profissionais revelaram que a situação de crise foi fortemente instigada pela ausência de protocolos de atendimento bem estabelecidos, delimitados e claramente repassados àqueles que atuavam na "linha de frente". Segundo suas percepções, o atendimento seria facilitado caso houvesse a instituição de protocolos e o seu repasse aos profissionais. 
Outro aspecto de destaque expresso no conteúdo referiu-se à presença de fragilidades e inadaptações na estrutura física, recursos materiais e humanos para o adequado atendimento aos pacientes e proteção dos trabalhadores, uma vez que a pandemia no Brasil espalhou-se rapidamente e houve pouco tempo de preparação para o enfrentamento.

O conteúdo revelou que, além da falta de preparo da UPA e do processo de trabalho, havia a necessidade de capacitação para as equipes de saúde. Destarte, tais situações vivenciadas nos serviços de PA no contexto da pandemia da Covid-19 expuseram a vulnerabilização dos profissionais, ao se evidenciar o acarretamento da contaminação de muitos profissionais de saúde, o consequente afastamento das atividades laborais e a vivência de sobrecarga no trabalho por aqueles que permaneciam na "linha de frente". Os participantes relataram esgotamento e exaustão física das equipes diante do contexto caótico.

Somado à situação de exaustão das equipes, os profissionais também relataram que a vivência da pandemia dentro da UPA foi marcada pela preocupação e medo, especialmente, de se contaminar e adoecer pela Covid-19, bem como de transmitir o vírus para os membros da sua família. Esse medo era intensificado pela falta de sensibilização e cooperação da população. Não obstante as orientações fornecidas pelos cientistas, epidemiologistas e outras categorias profissionais, amplamente divulgadas nas mídias, era perceptível que as recomendações e medidas preventivas não estavam/estão sendo cumpridas de forma rigorosa pela população.

Os achados empíricos estão ilustrados no Quadro 1.

Quadro 1 - Conteúdo temático das vivências de enfermeiros e médicos de unidades de Pronto Atendimento no enfrentamento da pandemia da Covid-19. Paraná, Brasil, 2020

(continua)

\begin{tabular}{|c|c|}
\hline \multicolumn{2}{|c|}{ Categoria temática 1: "A gente se sente esgotado": a vivência de enfermeiros e médicos } \\
\hline Unidade de sentido & Excerto das entrevistas \\
\hline $\begin{array}{l}\text { A Covid-19 enquanto uma } \\
\text { doença nova e as incertezas que } \\
\text { permeavam a prática profissional }\end{array}$ & $\begin{array}{l}\text { [...] no princípio houve bastante dificuldade no sentido de ser } \\
\text { uma doença nova, de não sabermos como ter o diagnóstico } \\
\text { correto, não ter teste disponível para o diagnóstico em larga } \\
\text { escala e, sobretudo, um tratamento definitivo da doença. (M7). } \\
\text { [...] ninguém sabia ao certo como a doença iria se comportar, } \\
\text { quais seriam os diagnósticos e como que ela evoluiria em termos } \\
\text { epidemiológicos. (M1). }\end{array}$ \\
\hline $\begin{array}{l}\text { Ausência de protocolos bem } \\
\text { estabelecidos na unidade para o } \\
\text { manejo clínico de pacientes com } \\
\text { a Covid-19 }\end{array}$ & $\begin{array}{l}\text { [...] no início da pandemia houve dificuldade por não haver um } \\
\text { protocolo correto estabelecido, qual paciente seria internado e } \\
\text { qual ficaria em isolamento, era necessária essa clareza para se } \\
\text { poder atender ao paciente. (M4). }\end{array}$ \\
\hline $\begin{array}{l}\text { Fragilidades e inadaptações } \\
\text { estruturais e de recursos } \\
\text { humanos para o enfrentamento } \\
\text { da pandemia }\end{array}$ & $\begin{array}{l}\text { [...] há dificuldades no atendimento desses pacientes com } \\
\text { suspeita [de Covid-19] por falta de infraestrutura, na verdade } \\
\text { nenhum lugar está preparado, a doença veio muito rápido em } \\
\text { nosso país. (M6). } \\
\text { [...] minha maior preocupação é com os EPIs e os cuidados com } \\
\text { o ambiente que a gente está exposta. (M2). } \\
\text { [...] acho que sofremos com a questão de organização do serviço, } \\
\text { faltou EPIs, faltou profissionais capacitados para enfrentar } \\
\text { os casos mais complexos, faltou a gente se sentir seguro. Se } \\
\text { eu for resumir, te digo que tudo que vivemos foi baseado em } \\
\text { dificuldades. (E6). }\end{array}$ \\
\hline $\begin{array}{l}\text { Problemáticas no processo de } \\
\text { trabalho em saúde e as carências } \\
\text { na capacitação das equipes } \\
\text { profissionais }\end{array}$ & $\begin{array}{l}\text { [...] faltou organização, faltou treinamento para a gente saber } \\
\text { como lidar com pacientes com sintomas da Covid-19, como } \\
\text { atender e encaminhar. (M3). } \\
\text { [...] a equipe não está preparada para essa situação, para esse } \\
\text { grau de pandemia. E os serviços não têm uma estrutura para } \\
\text { atender essa população, que deve ser atendida separadamente } \\
\text { dos demais. Sem organização e treinamento fica difícil. (M6). }\end{array}$ \\
\hline
\end{tabular}


Quadro 1 - Conteúdo temático das vivências de enfermeiros e médicos de unidades de Pronto Atendimento no enfrentamento da pandemia da Covid-19. Paraná, Brasil, 2020

\begin{tabular}{|c|c|}
\hline \multicolumn{2}{|c|}{ Categoria temática 1: "A gente se sente esgotado": a vivência de enfermeiros e médicos } \\
\hline $\begin{array}{l}\text { Vulnerabilização dos profissionais } \\
\text { à contaminação, o afastamento } \\
\text { do ambiente de trabalho e a } \\
\text { exaustão física }\end{array}$ & $\begin{array}{l}\text { A sobrecarga aconteceu, principalmente, porque trabalhamos sempre } \\
\text { no limite e agora tem muitos profissionais que acabam ficando } \\
\text { adoentados, ficando afastados, também tem o expressivo aumento do } \\
\text { número de pacientes, tudo isso sobrecarrega a equipe. (E4). } \\
\text { A gente se sente esgotado, cansado. A gente não pode negar que, } \\
\text { às vezes, bate o desespero, pois vem a exaustão e o esgotamento, } \\
\text { exaustão física e mental ao mesmo tempo. (M3). }\end{array}$ \\
\hline $\begin{array}{l}\text { Deflagração de medo e o } \\
\text { sentimento de preocupação dos } \\
\text { profissionais face à contaminação } \\
\text { e ao adoecimento pela Covid-19 } \\
\text { e de transmiti-la para outrem, } \\
\text { intensificados pela percepção da } \\
\text { falta de apoio da população }\end{array}$ & $\begin{array}{l}\text { [...] meu medo principal é de me contaminar, todos os dias vejo } \\
\text { notícias, principalmente, de profissionais da área que estão } \\
\text { internadas, que estão morrendo por conta da pandemia. (E1). } \\
\text { [...] o maior medo foi o de me contaminar, encarar as dificuldades e } \\
\text { aprender a lidar com esse medo [...] tenho receio de acabar trazendo } \\
\text { a doença para dentro da minha casa e, com isso, contaminar meus } \\
\text { familiares, esse tipo de medo ainda persiste. (M2). } \\
\text { [...] preocupação grande tivemos e ainda temos é na questão } \\
\text { de segurança da equipe e dos profissionais de saúde, para que } \\
\text { ninguém viesse a perder a vida [...] com a chegada desse novo vírus } \\
\text { fica evidente o medo e a preocupação diante do risco de infecção e a } \\
\text { possibilidade de transmitir a doença para nossos familiares. (E5). } \\
{[\text { [...] a população não está cumprindo com seu papel, que tem que }} \\
\text { se cuidar e nós tentamos fazer o melbor para eles. Mas, sem a } \\
\text { ajuda deles, fica mais difícil para nós trabalharmos. (M4). } \\
\text { [...] como é dificil as pessoas seguirem uma orientação básica, } \\
\text { não saia, use máscara, use álcool gel, são coisas que estão ao } \\
\text { alcance de todos, todos já sabem, já tem essas informações, mas } \\
\text { nem todo mundo cumpre. Por isso, existe insegurança de nossa } \\
\text { parte, a população não adere 10o\% às orientações que fazemos e } \\
\text { que a mídia também fornece o tempo todo. Parece que ninguém } \\
\text { está levando a sério! (E6). }\end{array}$ \\
\hline
\end{tabular}

Fonte: Elaboração própria.

\section{Categoria temática 2: Estratégias para enfrentar os percalços no contexto da pandemia}

Durante as entrevistas, os profissionais apontaram algumas estratégias empregadas para auxiliar no processo de enfrentamento dos desafios vivenciados durante a pandemia. Uma dessas estratégias era reflexo da preocupação de, possivelmente, contaminar os familiares, o que desencadeou o autoisolamento e as modificações nas relações de proximidade com sua rede social, aumentando o desgaste e o estresse dos profissionais.

Ao mesmo tempo em que a distância da família e dos amigos se reforçava como prática prudente dos profissionais para evitar a contaminação dos familiares, os entrevistados referiram a necessidade de apoio da família e dos amigos para enfrentar o estresse no trabalho e o momento de incertezas decorrente da pandemia, mesmo que por meio virtual.

A estratégia relatada para o enfrentamento da falta de protocolos clínicos, capacitação e insumos presente nas vivências profissionais, foi o compartilhamento de experiências com outros profissionais, para que possíveis falhas vivenciadas e identificadas no atendimento aos pacientes pudessem ser corrigidas. Outrossim, a busca por informações atualizadas e em fontes confiáveis foi uma estratégia profissional utilizada para manter-se atualizado sobre a evolução dos estudos acerca da doença. Esta se constituiu em uma ação individual e não algo organizado pelos serviços de saúde.

Os achados empíricos dessa categoria temática estão ilustrados no Quadro 2. 
Quadro 2 - Conteúdo temático das estratégias de enfrentamento da pandemia da Covid-19 engendradas por enfermeiros e médicos de unidades de Pronto Atendimento. Paraná, Brasil, 2020

\begin{tabular}{|c|c|}
\hline \multicolumn{2}{|c|}{ Categoria temática 2: Estratégias para enfrentar os percalços no contexto da pandemia } \\
\hline Unidade de sentido & Excerto das entrevistas \\
\hline $\begin{array}{l}\text { Autoisolamento e impactos da } \\
\text { distância da rede socioafetiva }\end{array}$ & $\begin{array}{l}\text { [...] eu não posso visitar o meu pai que é idoso, a minha família, } \\
\text { minhas irmãs que têm certa idade, amigos e conbecidos por estar } \\
\text { num ambiente em que atendo vários pacientes positivos para a } \\
\text { Covid-19. (E3). } \\
\text { [...] a distância é um impacto nas nossas vidas agora. Não poder } \\
\text { ficar perto da família, dos amigos, não podemos mais abraçar, } \\
\text { conversar, partilhar de uma refeição juntos, são momentos assim } \\
\text { que foram impactados pela pandemia. Sendo profissional da saúde, } \\
\text { podemos levar a doença para eles, temos que viver isolados. (E2). } \\
\text { [...] é isso que precisamos fazer, nos afastar. Isso nos deixa bastante } \\
\text { desanimados e preocupados. (M7). }\end{array}$ \\
\hline $\begin{array}{l}\text { Busca por suporte familiar e } \\
\text { de amigos para minimização } \\
\text { do estresse }\end{array}$ & $\begin{array}{l}\text { [...] eu tento me aproximar mais da minha famillia, dos meus } \\
\text { amigos, conversando através de ligação de videochamada à } \\
\text { distância. (E4). } \\
\text { [...] as pessoas que mais me apoiaram durante esse período da } \\
\text { pandemia foram minha esposa, meus filhos e meus pais. Esses são a } \\
\text { minha base. (M3). } \\
\text { [...] acho que meus familiares me deram um apoio muito grande, me } \\
\text { ajudaram a enfrentar essa fase estressante da minha carreira. (M5). } \\
\text { [...] uma colega de trabalho que eu gosto muito é quem mais me } \\
\text { apoia e a minha família à distância. Eu tive muito apoio dos } \\
\text { meus pais, principalmente da minha mãe que ela está sempre } \\
\text { preocupada em relação ao meu trabalho. (E7). }\end{array}$ \\
\hline $\begin{array}{l}\text { Ajuda profissional mútua na } \\
\text { busca pelo compartilhamento } \\
\text { de experiências e ideias }\end{array}$ & $\begin{array}{l}\text { [...] acho que a gente foi conversando, a gente foi cada um } \\
\text { contando a sua experiência de atendimento, a sua vivência com a } \\
\text { doença, e assim, alguns erros a gente foi aperfeiçoando. (M2). } \\
\text { [...] às vezes, conversava com um colega no serviço, era boa uma } \\
\text { estratégia para ir enfrentando os percalços e as dúvidas clínicas. (M3). } \\
\text { [...] minha esposa também é médica, também trabalha com pacientes } \\
\text { com suspeita da Covid-19, então a gente, no dia a dia ia conversando, } \\
\text { um apoiando e trocando experiências com o outro. (M1). }\end{array}$ \\
\hline $\begin{array}{l}\text { Ampliação do conhecimento } \\
\text { por meio da busca de } \\
\text { informações atuais e seguras }\end{array}$ & $\begin{array}{l}\text { [...] a gente procura sempre ler e se informar mais a respeito do tema, } \\
\text { fazendo um curso online, quando possível, para se atualizar sobre o } \\
\text { melhor tipo de atendimento. (E1). } \\
\text { [...] a principal forma de melhorar é se comunicar com colegas mais } \\
\text { experientes e sempre estar se atualizando sobre o que há de novo com } \\
\text { relação a doença, cada um busca sua fonte de informação confiável } \\
\text { e atualizada. (M7). }\end{array}$ \\
\hline
\end{tabular}

Fonte: Elaboração própria.

\section{Discussão}

Este estudo demonstra conteúdos empíricos que descrevem as vivências profissionais e pessoais de enfermeiros e médicos, durante a atuação na linha de frente contra a pandemia da Covid-19, no âmbito de UPAs de municípios de pequeno porte. Reforçou que o esgotamento estava presente entre profissionais de saúde e revelou algumas estratégias de enfrentamento dos percalços gerados pelo contexto pandêmico da Covid-19. Verifica-se que as estratégias de enfrentamento eram incipientes e foram pouco relatadas, sugerindo que, no momento do estudo, os profissionais ainda estavam mais propensos a identificar vivências de exaustão decorrentes do trabalho.

Entre os desafios vivenciados destaca-se o medo gerado pelo desconhecimento do diagnóstico, tratamento e prognóstico da Covid-19; 
a falta de protocolos para guiar o atendimento; a escassez de profissionais capacitados para atuar na linha de frente com os casos mais complexos; além da deficiência na estrutura física e organização dos serviços, especialmente no início da pandemia. Tais fatores foram citados como agravantes da exaustão física e psicológica dos profissionais.

Sabidamente, os desafios relatados decorrem do fato de a doença ser desconhecida. No que tange aos aspectos biológicos, por exemplo, a doença atinge as pessoas em diferentes níveis de complexidade e desencadeia quadros clínicos variados. Isso requer profissionais clinicamente experientes ou, no mínimo, bastante atentos para identificar casos de evolução de mal prognóstico, tomar decisões atempadas e tratar as possíveis complicações, reduzindo o risco de sequelas e óbito ${ }^{(12)}$. Nesse sentido, os achados do estudo em tela explicitam reflexões acerca de como enfermeiros e médicos têm conduzido a assistência e a produção do cuidado direcionado à Covid-19 diante dos desafios frequentes associados ao manejo clínico, às condutas terapêuticas, ao agir ético e à garantia da segurança e proteção profissional e para o paciente.

No concernente ao processo de trabalho na rede emergencial e hospitalar, outros desafios estiveram presentes. Por exemplo, estudo apontou que os maiores desafios se relacionaram à reorganização do atendimento, ampliação do número de leitos, abastecimento com equipamentos e insumos, capacitação dos profissionais e fornecimento de equipamentos de proteção individual e coletiva ${ }^{(13)}$. Tais aspectos foram relatados em estudo internacional como estratégias essenciais tanto para redução da infecção entre os profissionais de saúde como na redução da carga mental de estresse, ansiedade e sintomas relacionados à sobrecarga e ao medo durante o trabalho ${ }^{(14)}$.

Os relatos dos participantes deste estudo reforçaram a necessidade dos profissionais de se reinventar com a mudança repentina na rotina, com a falta de insumos e capacitação, bem como dificuldades no acesso aos EPIs. Esses fatores contribuíram ainda mais com a sobrecarga e a exaustão das equipes. À medida que a pandemia acelerou, o acesso aos EPIs tornou-se uma preocupação constante, especialmente para os profissionais atuantes nas UPAs, local onde o risco é reconhecidamente maior devido aos procedimentos invasivos que liberam no ambiente aerossóis potencialmente transmissores do vírus ${ }^{(12)}$. A escassez de EPI foi observada em diversas instituições de outros países, não somente nas brasileiras. Destaca-se que a manutenção de EPI nos hospitais deve ser uma política de Estado, na qual os governos precisam se mobilizar para que a indústria nacional responda ao desafio. Entretanto, observou-se uma especulação dos preços, que tiveram aumento associado ao desabastecimento do mercado.

Para além, devido à ausência de um protocolo único a ser seguido, a falta de EPI e a falta de capacitação sobre a forma correta de paramentação, desparamentação e descarte fizeram com que os trabalhadores da saúde ficassem mais vulneráveis à contaminação ${ }^{(12,14)}$. Face a esse contexto, enfatiza-se que, mais do que uma modificação no fazer profissional de enfermeiros e médicos que atuam nas unidades de PA, a pandemia expôs uma desagregação para a manutenção do processo de trabalho e agudizou problemáticas de precarização e de desmantelamento dos serviços públicos de saúde e do próprio SUS já, historicamente, existentes nesses $\operatorname{espaços~}^{(2)}$.

Frente a essas vivências profissionais, há que se destacar que os achados despontam para uma análise sobre como as UPAs de municípios de pequeno porte foram afetadas pela pandemia e de que modo se organizaram para o enfrentamento da crise sanitária. Esses resultados, quando expressos por meio de estudos, mostram-se como importantes subsídios para instrumentalizar gestores, apoiadores técnicos, agentes de implementação de políticas públicas e profissionais de saúde, e incrementa as ações focadas no planejamento e na execução das estratégias programáticas em saúde em contextos pandêmicos.

No enfrentamento da situação, os profissionais revelaram estratégias tomadas de forma 
individual, conduzidas, na maior parte das vezes, por seus conhecimentos e experiências pessoais. Frente ao medo da contaminação de si próprio, dos familiares e amigos relatado pelos participantes, muitos optaram por realizar o autoisolamento, o que contribuiu para o sofrimento mental dos trabalhadores. Sabidamente, profissionais de saúde são particularmente mais susceptíveis e constituem grupo de risco para a Covid-19 por estarem expostos diretamente aos pacientes infectados ${ }^{(4,14)}$.

Em consonância com outros achados, o medo de ser infectado, a proximidade com o sofrimento e/ou morte de pacientes, além da angústia dos familiares, a falta de suprimentos médicos, informações incertas, solidão e preocupação com os entes queridos também foram aspectos evidenciados em estudo sobre as experiências de enfermeiros durante a pandemia da Covid$19^{(15)}$. Além disso, é importante refletir sobre o impacto gerado pelo cotidiano epidêmico às vivências profissionais cotidianas de enfermeiros e médicos no fazer e no exercício profissional nas unidades de PA, que revelam fragilizações e vulnerabilidades daqueles que produzem cuidado nesses espaços, em que pese um contexto em que se observam capacitações profissionais tardias, exposição das equipes, afastamento do trabalho, adoecimento e óbito de enfermeiros e médicos.

A exposição constante e o medo de ser infectado são reafirmados em números. Milhares de profissionais de saúde foram afastados de suas atividades laborais, durante o ano de 2020, por terem adquirido a infecção. Verifica-se que sete mil profissionais de saúde no mundo morreram em decorrência da infecção pelo coronavírus, sendo o Brasil, o terceiro país com mais mortes de profissionais da saúde durante a pandemia ${ }^{(16)}$. O Conselho Federal de Enfermagem (COFEN) afirmou em documento público que o Brasil responde por um terço do total de mortes pela Covid-19 entre os profissionais dessa categoria $^{(17)}$. Desse modo, frente aos resultados do estudo em tela, cabe inferir que no contexto da pandemia, o acesso de pacientes com necessidades clínicas de saúde em caráter de urgência e emergência, em especial, em municípios de pequeno porte, foi consideravelmente elevado. Entretanto, as equipes profissionais desses serviços não estavam completamente preparadas e protegidas para atuar frente ao desconhecido, e em uma clínica ainda pouco padronizada para a condução assistencial. Isso, em parte, explica a exaustão profissional.

Além disso, é importante destacar que, num contexto de estresse e incertezas, inclusive sobre os modos de atuação profissional no âmbito das unidades de PA, distintas necessidades tiveram que ser supridas por enfermeiros e médicos, a saber: incorporação das diretrizes clínicas, consumo de evidências científicas e a distinção de fake news, medidas de biossegurança, normas e rotinas assistenciais, e outras especificidades de uma unidade de urgência e emergência. Essas necessidades podem ter contribuído para a exaustão física e mental dos profissionais.

A carência de protocolos técnicos definidos, a ausência de acesso à equipe de psicologia, bem como as dificuldades de resiliência e de habilidade de gestão de crise configuram-se como fatores estressores e contribuem para o sofrimento e a angústia moral dos profissionais de saúde. Comumente, o sofrimento e angústia moral são elevados entre os profissionais que desenvolvem cuidados críticos diretos a pacientes graves. Durante a pandemia da Covid-19, tal fato tem se tornado mais frequente diante da quantidade de pacientes graves, alavancado com a sobrecarga dos serviços de saúde, semelhante a situações de guerra, levando os profissionais a se tornarem as novas vítimas da pandemia ${ }^{(18)}$.

Nesse sentido, as evidências mostram que as equipes da "linha de frente" apresentam exaustão física, mental, dificuldade na tomada de decisão e ansiedade pela dor de perder muitos pacientes e colegas de trabalho, somado à possibilidade de transmissão da doença aos familiares, especialmente para aqueles que cuidam dos pais ou filhos pequenos $^{(19)}$. O temor em contaminar familiares é um risco psicossocial relevante, portanto, é fundamental que sejam garantidas medidas de atenuação desse risco, como a oferta da assistência médica e apoio psicológico, oferta 
de alojamentos fora do local de trabalho, por exemplo $^{(12)}$.

Algumas instituições têm disponibilizados alojamentos em hotéis para profissionais de saúde que desejam descansar fora de sua residência como medida de segurança para seus familiares. Isso foi reconhecido como uma estratégia de sucesso na China, que também implementou o transporte seguro para o deslocamento dos trabalhadores até o local de trabalho ${ }^{(20)}$. Entretanto, estudos apontam que, mesmo com intenso treinamento, é comum que os enfermeiros descuidem da sua exposição enquanto cuidam de pacientes, especialmente quando estão exaustos, após longas jornadas de trabalho, aumentando o risco de contaminação ${ }^{(15,19)}$.

Publicações científicas e televisivas ao longo do ano de 2020 mostraram que as equipes de saúde têm passado por sofrimento e exaustão devido a pandemia da Covid-19 ${ }^{(21-22)}$. Assim, é ético e humanamente relevante o acompanhamento psicológico e psiquiátrico dos profissionais, tendo em vista que o cuidado em saúde mental favorece a melhor atuação no local de trabalho e a ausência desse apoio reduzirá o potencial de cuidado, aumentando as chances de afastamento, contaminações, mortes e outras consequências posteriores ${ }^{(21-22)}$.

O apoio familiar e de amigos, o acompanhamento com equipe multiprofissional por meio do uso da telemedicina, bem como a capacitação dos profissionais foram estratégias evidenciadas como formas de enfrentamento dos desafios da pandemia com impacto na saúde mental dos profissionais ${ }^{(23)}$. Além disso, a compreensão acerca das medidas de prevenção e a contenção da proliferação da transmissão pela população leva um efeito positivo nas respostas psicológicas dos profissionais de saúde e da própria população em geral $^{(24)}$.

Nesse sentido, faz-se necessário que as autoridades de saúde, em conjunto com os governantes, realizem atualizações e comunicações sobre as características e consequências da pandemia de modo a impedir a divulgação de notícias falsas, assim como promover capacitações continuadas para os profissionais atuantes na linha de frente. O programa "Brasil conta comigo - profissionais de saúde", lançado pelo Ministério da Saúde ${ }^{(25)}$, direcionou a capacitação e o cadastramento de profissionais da área para o enfrentamento à pandemia da Covid-19. Entretanto, ainda é preciso ampliar e melhorar tais estratégias, para auxiliar e apoiar os profissionais nesse momento, especialmente aqueles inseridos no interior do país e em municípios de pequeno porte.

Frente aos resultados arrolados, é preciso ponderar que esta investigação possui limitações, entre elas, destaca-se que a coleta de dados foi realizada nas dependências da unidade de PA, de modo que, possivelmente, alguns entrevistados estivessem preocupados com o retorno ao trabalho, limitando suas respostas. Outro ponto relaciona-se ao fato de a pesquisa ter sido realizada em número limitado de instituições e com número limitado de entrevistados, circunscrevendo os resultados ao contexto em análise. Portanto, recomenda-se que sejam realizados estudos futuros sobre a temática, em cenários que favoreçam a coleta de dados e em regiões diversas do país, para que se obtenha uma perspectiva ampliada a respeito das repercussões da pandemia da Covid-19 na vida dos profissionais de saúde atuantes em UPAs.

\section{Considerações finais}

As vivências profissionais de enfermeiros e médicos que atuam em UPA foram marcadas por desafios relacionados ao fato de a Covid-19 ser uma doença nova, o que dificultava o diagnóstico: a falta de protocolo institucional que direcionasse concretamente a prática; a falta de estrutura física, material e de recursos humanos para o adequado atendimento; a falta de capacitação da equipe; o medo e a preocupação de se contaminar e contaminar a família; e ainda, a dificuldade para sensibilizar a população sobre a adoção de medidas preventivas.

Para mitigar as problemáticas e dificuldades profissionais vivenciadas no cotidiano das UPAs, enfermeiros e médicos empregaram diferentes estratégias de enfrentamento. Destacaram-se 
o autoisolamento como medida preventiva, o apoio de outros profissionais de saúde no compartilhamento de informações e vivências, a busca pelo suporte familiar e de amigos, mesmo que à distância, e a busca individual e constante por informações atualizadas sobre a doença e seu tratamento.

\section{Colaborações:}

1 - concepção, projeto, análise e interpretação dos dados: Mayckel da Silva Barreto, Sonia Silva Marcon, Anderson Reis de Sousa, Rafaelly de Cássia Nogueira Sanches, Hellen Pollyanna Mantelo Cecilio, Dulcineia Martins Pinto e Renata Tresco de Oliveira;

2 - redação do artigo e revisão crítica relevante do conteúdo intelectual: Mayckel da Silva Barreto, Sonia Silva Marcon, Anderson Reis de Sousa, Rafaelly de Cássia Nogueira Sanches, Hellen Pollyanna Mantelo Cecilio, Dulcineia Martins Pinto e Renata Tresco de Oliveira;

3 - aprovação final da versão a ser publicada: Mayckel da Silva Barreto, Sonia Silva Marcon, Anderson Reis de Sousa, Rafaelly de Cássia Nogueira Sanches, Hellen Pollyanna Mantelo Cecilio, Dulcineia Martins Pinto e Renata Tresco de Oliveira.

\section{Referências}

1. Kucharski AJ, Russel TW, Diamond C, Liu Y, Edmunds J, Funk S, et al. Early dynamics of transmission and control of COVID-19: a mathematical modelling study. Lancet Infect Dis. 2020;20(5):553-8. DOI: https://doi.org/10.1016/ S1473-3099(20)30144-4

2. Cueto M. Coronavirus y el blog de História, Ciências, Saúde - Manguinhos. Hist cienc saude-Manguinhos. 2020;27(2):327-30. DOI: http:// dx.doi.org/10.1590/s0104-59702020000200001

3. Daumas RP, Azevedo e Silva G, Tasca R, Leite IC, Brasil P, Greco DB, et al. O papel da atenção primária na rede de atenção à saúde no Brasil: limites e possibilidades no enfrentamento da COVID-19. Cad Saúde Pública. 2020;36(6):e00104120. DOI: https://doi. org/10.1590/0102-311X00104120
4. Helioterio MC, Lopes FQRS, Sousa CC, Souza FO, Pinho PS, Sousa FNF, et al. Covid-19: Por que a proteção de trabalhadores e trabalhadoras da saúde é prioritária no combate à pandemia?. Trab educ saúde. 2020;18(3): e00289121. DOI: https://doi.org/10.1590/1981-7746sol00289

5. Bravo K, Gamarra M, Gonzales S, Lecca D, Martínez A, Mendonza $M$, et al. Nivel de conocimientos sobre la influenza a H1N1 en los trabajadores de salud del Hospital Nacional Arzobispo Loayza, Perú. Rev Peru Epidemiol [Internet]. 2009 [cited 2020 Jul 31];13(2):1-7. Available from: https://www.redalyc.org/ pdf/2031/203120363003.pdf

6. Shiao JSC, Koh D, Lo LH, Lin MK, Guo YL. Factors Predicting Nurses' Consideration of Leaving their Job During the SARS Outbreak. Nurs Ethics. 2007;14(1):5-17. DOI: https://doi. org/10.1177/0969733007071350

7. Kim JS, Choi JS. Factors Influencing Emergency Nurses' Burnout During an Outbreak of Middle East Respiratory Syndrome Coronavirus in Korea. Asian Nurs Res. 2016;10(4):295-9. DOI: 10.1016/j. anr.2016.10.002

8. Teixeira AC, Pereira R, Silva JAP. Facing COVID-19: experience report from a Portuguese intensive care unit. Rev Parana Enferm. 2020 [cited 2021 Jan 2];3(1):82-90. Available from: http://seer.fafiman.br/index.php/REPEN/article/ view/599/553

9. Souza e Souza LP, Souza AG. Enfermagem brasileira na linha de frente contra o novo Coronavírus: quem cuidará de quem cuida? J Nurs Health. 2020; 10(4):e20104005. DOI: https:// doi.org/10.15210/jonah.v10i4.18444

10. Kim Y. Nurses' experiences of care for patients with Middle East respiratory syndrome-coronavirus in South Korea. Am J Infect Control. 2018;46(7):781-7. DOI: https://doi. org/10.1016/j.ajic.2018.01.012

11. Bardin L. Análise de conteúdo. Lisboa: Edições 70; 2016.

12. Medeiros EAS. A luta dos profissionais de saúde no enfrentamento da COVID-19. Acta Paul Enferm. 2020;33:e-EDT20200003. DOI: https://doi.org/10.37689/acta-ape/2020edt 0003

13. Lipsitch M, Swerdlow DL, Finelli L. Defining the Epidemiology of Covid-19 - Studies Needed. 
N Engl J Med. 2020;382:1194-6. DOI: 10.1056/ NEJMp2002125

14. Cattelan AM, Sasset L, Di Meco E, Cocchio S, Barbaro F, Cavinato S, et al. An Integrated Strategy for the Prevention of SARS-CoV-2 Infection in Healthcare Workers: A Prospective Observational Study. Int J Environ Res Public Health. 2020;17(16):5785. DOI: https://doi.org/ 10.3390/ijerph17165785

15. Huang L, Lin G, Tang L, Yu L, Zhou Z. Special attention to nurses' protection during the COVID-19 epidemic. Crit Care. 2020;24(1):120. DOI: https://doi.org/10.1186/s13054-020-2841-7

16. Amnesty International. Global: Amnesty analysis reveals over 7,000 health workers have died from COVID-19 [Internet]. Londres (UK); 2020 [cited 2021 Jan 19]. Available from: https://www. amnesty.org/en/latest/news/2020/09/amnestyanalysis-7000-health-workers-have-died-fromcovid19

17. Conselho Federal de Enfermagem. Brasil é o país com mais mortes de enfermeiros por Covid-19 no mundo [Internet]. Brasília (DF); 2020 [cited 2021 Jan 19]. Available from: http://www.cofen.gov.br/ brasil-e-o-pais-com-mais-mortes-de-enfermeirospor-covid-19-no-mundo-dizem-entidades_80181. html

18. Borges LM, Barnes SM, Farnsworth JK, Bahraini NH, Brenner LA. A commentary on moral injury among health care providers during the COVID-19 pandemic. Psychol Trauma. 2020;12(S1):S138-S140. DOI: http://dx.doi.org/10. 1037/tra0000698

19. Prado AD, Peixoto BC, Silva AMB, Scalia LAM. A saúde mental dos profissionais de saúde frente à pandemia do COVID-19: uma revisão integrativa. Rev Eletr Acervo Saúde. 2020;(46):e4128. DOI: https://doi.org/10.25248/ reas.e 4128.2020

20. Zhang Z, Liu S, Xiang M, Li S, Zhao D, Huang $C$, et al. Protecting healthcare personnel from 2019-nCoV infection risks: lessons and suggestions. Front Med. 2020;14(2):229-31. DOI: 10.1007/s11684-020-0765-X

21. Carvalho PMM, Moreira MM, Oliveira MNA, Landim JMM, Rolim Neto ML. The psychiatric impact of the novel coronavirus outbreak. Psychiatry Res. 2020;286:112902. DOI: 10.1016/j. psychres.2020.112902

22. Li Z, Ge J, Yang M, Feng J, Qiao M, Jiang R, et al. Vicarious traumatization in the general public, members, and non-members of medical teams aiding in COVID-19 control. Brain, Behav Immun. 2020;88:916-9. DOI: https://doi. org/10.1016/j.bbi.2020.03.007

23. Ho CS, Chee CY, Ho RC. Mental Health Strategies to Combat the Psychological Impact of Coronavirus Disease 2019 (COVID-19) Beyond Paranoia and Panic. Ann Acad Med Singap [Internet]. 2020 [cited 2021 Jan 19];49(3):155-60. Available from: https://www.annals.edu.sg/pdf/ 49VolNo3Mar2020/V49N3p155.pdf

24. Wang C, Pan R, Wan X, Tan Y, Xu L, Ho CS, et al. Immediate Psychological Responses and Associated Factors during the Initial Stage of the 2019 Coronavirus Disease (COVID-19) Epidemic among the General Population in China. Int $\mathrm{J}$ Environ Res Public Health. 2020;17(5):1729. DOI: https://doi.org/10.3390/ijerph17051729

25. Brasil. Ministério da Saúde. Portaria 639, de 31 de Março de 2020. Dispõe sobre a Ação Estratégica "O Brasil Conta Comigo - Profissionais da Saúde", voltada à capacitação e ao cadastramento de profissionais da área de saúde, para o enfrentamento à pandemia do coronavírus (COVID-19) [Internet]. Diário Oficial da União. Brasília (DF); 2020 [cited 2121 Jan 19]. Available from: https://www.in.gov.br/en/web/dou/-/portarian-639-de-31-de-marco-de-2020-250847738

Recebido: 13 de fevereiro de 2021

Aprovado: 18 de maio de 2021

Publicado: 22 de julho de 2021

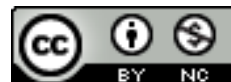

A Revista Baiana de Enfermagem utiliza a Licença Creative Commons - Atribuição-NãoComercial 4.0 Internacional. https://creativecommons.org/licenses/by-nc/4.0/ Este artigo é de acesso aberto distribuído sob os termos da Licença Creative Commons (CC BY-NC). Esta licença permite que outros remixem, adaptem e criem a partir do seu trabalho para fins não comerciais. Embora os novos trabalhos tenham de lhe atribuir o devido crédito e não possam ser usados para fins comerciais, os usuários não têm de licenciar esses trabalhos derivados sob os mesmos termos. 